\title{
Subclinical Hypothyroidism Affects the Long-Term Outcomes of Patients Who Undergo Coronary Artery Bypass Grafting Surgery but Not Heart Valve Surgery
}

\author{
Hana Kim ${ }^{1,2}$, Sung Hye Kong ${ }^{1}$, Jae Hoon Moon ${ }^{3}$, Sang Yoon Kim ${ }^{4}$ Kay-Hyun Park ${ }^{4}$, Jun Sung Kim ${ }^{4}$ Joong Haeng Choh $^{4}$ \\ Young Joo Park ${ }^{1}$, Cheong Lim ${ }^{4}$ \\ ${ }^{1}$ Department of Internal Medicine, Seoul National University College of Medicine, Seoul; ${ }^{2}$ Department of Internal Medicine, \\ Ilsan Cha Medical Center, Goyang; Departments of ${ }^{3}$ Internal Medicine, ${ }^{4}$ Thoracic and Cardiovascular Surgery, Seoul National \\ University Bundang Hospital, Seongnam, Korea
}

Background: The aim of this study was to determine the associations between subclinical hypothyroidism (SCH) and long-term cardiovascular outcomes after coronary artery bypass grafting (CABG) or heart valve surgery (HVS).

Methods: We retrospectively reviewed and compared all-cause mortality, cardiovascular mortality, and cardiovascular events in 461 patients who underwent CABG and 104 patients who underwent HVS.

Results: During a mean \pm standard deviation follow-up duration of $7.6 \pm 3.8$ years, there were 187 all-cause deaths, 97 cardiovascular deaths, 127 major adverse cardiovascular events (MACE), 11 myocardial infarctions, one unstable angina, 70 strokes, 30 hospitalizations due to heart failure, 101 atrial fibrillation, and 33 coronary revascularizations. The incidence of all-cause mortality after CABG was significantly higher in patients with $\mathrm{SCH}(n=36,55.4 \%)$ than in euthyroid patients $(n=120,30.3 \%)$, with a hazard ratio of 1.70 (95\% confidence interval, 1.10 to $2.63 ; P=0.018)$ after adjustment for age, sex, current smoking status, body mass index, underlying diseases, left ventricular dysfunction, and emergency operation. Interestingly, low total triiodothyronine (T3) levels in euthyroid patients who underwent $\mathrm{CABG}$ were significantly associated with increased risks of all-cause mortality, cardiovascular mortality, and MACE, but those associations were not observed in HVS patients. Both free thyroxine and thyroid-stimulating hormone levels in euthyroid patients were not related with any cardiovascular outcomes in either the CABG or HVS group.

Conclusion: $\mathrm{SCH}$ or low total $\mathrm{T} 3$ might be associated with a poor prognosis after $\mathrm{CABG}$, but not after HVS, implying that preoperative thyroid hormonal status may be important in ischemic heart disease patients.

Keywords: Cardiovascular diseases; Heart valve diseases; Cause of death; Prognosis; Hypothyroidism; Thyrotropin; Triiodothyronine

\section{INTRODUCTION}

Subclinical hypothyroidism ( $\mathrm{SCH})$, which is defined as elevated

Received: 22 December 2019, Revised: 3 March 2020, Accepted: 6 April 2020 Corresponding authors: Young Joo Park

Department of Internal Medicine, Seoul National University College of Medicine, 101 Daehak-ro, Jongno-gu, Seoul 03080, Korea

Tel: +82-2-2072-4183, Fax: +82-2-764-2199, E-mail: yjparkmd@snu.ac.kr

Cheong Lim

Department of Thoracic and Cardiovascular Surgery, Seoul National University

Bundang Hospital, 82 Gumi-ro 173beon-gil, Bundang-gu, Seongnam 13620,

Korea

Tel: +82-31-787-7139, Fax: +82-31-787-4050, E-mail: mluemoon@snu.ac.kr serum thyroid-stimulating hormone (TSH) levels with normal free thyroxine (fT4) levels, is a very prevalent condition, especially in iodine-rich areas $[1,2]$. Therefore, concerns have been

\section{Copyright $\odot 2020$ Korean Endocrine Society}

This is an Open Access article distributed under the terms of the Creative Commons Attribution Non-Commercial License (https://creativecommons.org/ licenses/by-nc/4.0/) which permits unrestricted non-commercial use, distribution, and reproduction in any medium, provided the original work is properly cited. 
raised regarding the health effects of $\mathrm{SCH}$, and a number of studies have shown an association between $\mathrm{SCH}$ and adverse clinical outcomes, especially an increased risk of cardiovascular morbidity or mortality [3-6]. Nonetheless, some inconsistent results have been reported, showing no correlation between TSH levels and cardiovascular outcomes [3,7-11]. Despite these discrepancies, a meta-analysis showed a modest increase in the risks of cardiovascular disease (CHD) and mortality in $\mathrm{SCH}$ subjects, particularly among subjects with higher TSH levels $[12,13]$. Recently, we also demonstrated a clear association between higher TSH levels and cardiovascular risk among individuals with high cardiovascular risk in both a population-based prospective study [14] and a meta-analysis [15].

Therefore, morbidity or mortality after cardiac surgery could be influenced by the presence of $\mathrm{SCH}$, especially in patients with high cardiovascular risk. In particular, $\mathrm{SCH}$ might influence the clinical outcomes of patients who undergo coronary artery bypass grafting $(\mathrm{CABG})$, because their cardiovascular risk is very high. In this regard, we previously reported that preoperative $\mathrm{SCH}$ patients showed a higher incidence of postoperative atrial fibrillation (Afib) than their euthyroid counterparts [16]. We also have shown that the incidence of postoperative coronary revascularization and non-target lesion revascularization was significantly higher in patients with $\mathrm{SCH}$ than in those with euthyroid status after 8.2 years of follow-up of a cohort [17]. These results emphasize the possible importance of $\mathrm{SCH}$ for predicting surgical outcomes in these patients. However, in that study, we did not observe significant differences in all-cause or cardiovascular mortality in $\mathrm{SCH}$ patients after CABG. Moreover, we did not confirm whether the poor outcomes of $\mathrm{SCH}$ patients were specific to CABG or would be similar after other cardiac surgical procedures in patients whose cardiovascular risk is not as high. A previous study showed increased postoperative Afib in SCH patients after aortic valve replacement surgery [18], although they did not present long-term outcomes after surgery.

To clarify whether the influence of preoperative $\mathrm{SCH}$ on surgical outcomes after cardiac surgery is limited to patients with high cardiovascular risk and whether it makes a difference in mortality after surgery, we analyzed long-term outcomes in a large number of patients who underwent $\mathrm{CABG}$ in addition to the previous cohort, and compared their long-term prognosis to that of patients who underwent heart valve surgery (HVS).

\section{METHODS}

\section{Study subjects}

We retrospectively reviewed 476 patients who underwent CABG and 109 patients who underwent HVS at Seoul National University Bundang Hospital (SNUBH) from July 2003 to December 2009. The 476 CABG patients included 233 patients from our previous cohort (195 euthyroid and $38 \mathrm{SCH}$ ) and 228 new patients (200 euthyroid and $28 \mathrm{SCH}$ ) who subsequently underwent $\mathrm{CABG}$. Fifteen patients who underwent $\mathrm{CABG}$ and 5 patients who underwent HVS were excluded due to a lack of clinical data or loss to follow-up. Finally, 395 euthyroid and $66 \mathrm{SCH}$ patients were included in the CABG group, while 87 euthyroid and $17 \mathrm{SCH}$ patients were included in the HVS group. Their thyroid function was measured within 1 month before surgery, and no treatment was performed if their fT4 level was normal. Participants were clustered by preoperative TSH and fT4 levels. The study was approved by the Institutional Review Board of SNUBH (B1901/516-105). There was no requirement for informed consent due to the retrospective nature of the study.

\section{Medical histories and anthropometric measurements}

We confirmed patients' underlying diseases, including hypertension, dyslipidemia, diabetes mellitus, cerebrovascular disease, and current smoking via their medical records. We defined chronic kidney disease according to the National Kidney Foundation criteria, and Afib was diagnosed by 12-lead electrocardiography (ECG) according to the American College of Cardiology (ACC), American Heart Association (AHA), and European Society of Cardiology (ESC) guidelines published in 2006. We measured patients' height and body weight using a scale and wall-fixed extensimeter.

\section{Cardiac surgery procedures and laboratory assessment}

The CABG procedures were conducted by four surgeons (Drs. K.H.P., J.S.K., J.H.C., and C.L.) as described previously $[16,19]$. In CABG, grafting of the left internal thoracic artery to the left anterior descending artery was performed whenever possible. Other vessels were revascularized with the right internal thoracic artery, radial artery, gastroepiploic artery, or saphenous vein. Off-pump, on-pump beating, or a conventional strategy for cardiopulmonary bypass (CPB) was chosen in accordance with the patient's condition. HVS procedures were performed under $\mathrm{CPB}$ support utilizing the ascending aorta and bicaval cannulation. Most of the time, moderate hypothermia $\left(28^{\circ} \mathrm{C}\right.$ to $\left.32^{\circ} \mathrm{C}\right)$ was induced during aortic cross-clamping. Ei- 
ther cold blood or crystalloid cardioplegia was used.

Blood samples were collected at the outpatient clinic within 1 month before surgery or at the admission time for urgent or emergent surgery. Serum TSH, fT4, and total triiodothyronine (T3) levels were measured by immunoradioassay using commercial kits (TSH, CIS Biointernational, Gif-sur-Yvette, France; fT4 and total T3, DiaSorin S.p.A, Saluggia, Italy). Euthyroid status was defined as normal TSH (range, 0.4 to $4.0 \mathrm{mIU} / \mathrm{L}$ ) and fT4 (range, 0.7 to $1.8 \mathrm{ng} / \mathrm{dL}$ ) levels without any thyroidal drug. $\mathrm{SCH}$ was defined as a TSH level over $4.0 \mathrm{mIU} / \mathrm{L}$ with a fT4 level in the normal range. Serum total cholesterol, triglyceride, and high-density lipoprotein cholesterol levels were measured using an autoanalyzer (Hitachi 747, Hitachi Ltd., Tokyo, Japan). Low-density lipoprotein cholesterol levels were estimated as total cholesterol-[high-density cholesterol+(triglycerides/5)] [20]. Colorimetry with an HST 403XE cell counter (Sysmex Corp., Kobe, Japan) was used to measure hemoglobin levels. Creatinine and high-sensitivity C-reactive protein were measured by an autoanalyzer (Hitachi 747).

\section{Follow-up and long-term prognosis}

The patients were followed at 1 to 2 weeks and 1, 3, and 6 months after the operation, and every 6 to 12 months later. The follow-up schedule depended on each patient's medical and social condition. Long-term prognosis was defined in terms of the complications that occurred within 3 months after surgery. We collected data related with all-cause death and major adverse cardiovascular events (MACEs), comprising cardiovascular death, nonfatal myocardial infarction (MI), nonfatal stroke, or hospitalization for heart failure. Cardiovascular death was defined as mortality due to postoperative arrhythmia, acute MI, cardiogenic shock, heart failure, or cerebrovascular events. Data were also collected on postoperative outcomes, such as Afib, unstable angina, and coronary revascularization. We confirmed all-cause and cardiovascular death by reviewing both patients' electronic medical records and the mortality records of the $\mathrm{Na}$ tional Statistical Office in South Korea in April 2019. Acute MI was diagnosed by 12-lead ECG and elevated cardiac biomarkers with typical angina symptoms. Unstable angina was defined by newly appearing typical chest pain or pain aggravation and positive ECG findings without cardiac biomarker elevation. Hospitalization due to heart failure was defined as an admission for at least 12 hours with typical signs of heart failure (dyspnea, orthopnea, pulmonary, and peripheral edema, jugular venous distension, pulmonary effusion on X-ray, or related results on echocardiography). Postoperative Afib was confirmed by the patients' ECG within 3 months after surgery. We defined each postoperative event based on the ACC, AHA, and ESC practice guidelines [21-23]. Both CABG and percutaneous coronary intervention were included as postoperative coronary revascularization.

\section{Statistical analysis}

Continuous variables are presented as mean \pm standard deviation, while categorical variables are presented as numbers and percentages. The patients' baseline characteristics were analyzed using the Student $t$ test or chi-square test, as appropriate. Cox proportional hazards regression models were used to estimate hazard ratio (HR) and 95\% confidence interval (CI) for all-cause mortality, cardiovascular mortality, MACE, MI, unstable angina, stroke, hospitalization for heart failure, Afib, and coronary revascularization. We calculated multivariate HRs by adjusting for age, sex, body mass index (BMI), smoking status, left ventricular dysfunction, emergency operation, and underlying diseases such as diabetes, hypertension, dyslipidemia, and chronic kidney disease. Survival time was estimated to have lasted from the date of surgery to death or the last follow-up date. To compare survival according to thyroid function status before surgery, Kaplan-Meier curves were used and $P$ values were calculated using the log-rank test. A $P<0.05$ was considered to indicate statistical significance. All analyses were performed using SPSS version 25.0 (IBM Corp., Armonk, NY, USA).

\section{RESULTS}

\section{Baseline characteristics}

The baseline characteristics of the study subjects are shown in Table 1. Patients' mean age was higher in the CABG group than in the HVS group (65.9 \pm 9.9 years vs. $60.7 \pm 12.3$ years), and the proportion of male patients was also higher (71.4\% vs. $45.2 \%$ ). In addition, $\mathrm{CABG}$ patients showed a higher frequency of conditions related to cardiovascular risk, including a higher BMI and more underlying diseases such as hypertension, diabetes mellitus, dyslipidemia and chronic kidney disease, than the patients who underwent HVS. However, more current smokers were included in the HVS group than in the CABG group. There were no significant differences in TSH and total T3 levels between the two groups, while fT4 levels were significantly higher (although within the normal range) in the HVS group than in the CABG group. Within the CABG and HVS groups, there were no significant differences in mean age, sex, or BMI between euthyroid and $\mathrm{SCH}$ patients. Furthermore, within the 


\begin{tabular}{|c|c|c|c|c|c|c|c|c|c|}
\hline \multirow[b]{2}{*}{ Variable } & \multicolumn{4}{|c|}{ Patients with IHD } & \multicolumn{4}{|c|}{ Patients with VHD } & \multirow[b]{2}{*}{$P$ value $^{\mathrm{a}}$} \\
\hline & $\begin{array}{c}\text { Total } \\
(n=461)\end{array}$ & $\begin{array}{c}\text { Euthyroid } \\
(n=395)\end{array}$ & $\begin{array}{c}\mathrm{SCH} \\
(n=66)\end{array}$ & $P$ value & $\begin{array}{c}\text { Total } \\
(n=104)\end{array}$ & $\begin{array}{l}\text { Euthyroid } \\
(n=87)\end{array}$ & $\begin{array}{c}\mathrm{SCH} \\
(n=17)\end{array}$ & $P$ value & \\
\hline Age, yr & $65.9 \pm 9.9$ & $65.8 \pm 9.8$ & $66.4 \pm 10.4$ & 0.651 & $60.7 \pm 12.4$ & $60.7 \pm 12.3$ & $60.7 \pm 12.8$ & 0.994 & $<0.001$ \\
\hline Male sex & $329(71.4)$ & $288(72.9)$ & $41(62.1)$ & 0.073 & $47(45.2)$ & $43(49.4)$ & $4(23.5)$ & 0.064 & $<0.001$ \\
\hline BMI, $\mathrm{kg} / \mathrm{m}^{2}$ & $24.7 \pm 3.0$ & $24.7 \pm 3.0$ & $24.7 \pm 3.0$ & 0.996 & $23.9 \pm 3.5$ & $23.9 \pm 3.6$ & $24.1 \pm 3.2$ & 0.824 & $<0.050$ \\
\hline Hypertension & $346(75.1)$ & $293(74.2)$ & $53(80.3)$ & 0.219 & $32(30.8)$ & $29(33.3)$ & $3(17.6)$ & 0.258 & $<0.001$ \\
\hline Diabetes & $243(52.7)$ & $205(51.9)$ & $38(57.6)$ & 0.350 & $11(10.6)$ & $9(10.3)$ & $2(11.8)$ & 1.000 & $<0.001$ \\
\hline Dyslipidemia & $181(39.3)$ & $157(39.8)$ & $24(36.4)$ & 0.592 & $4(3.8)$ & $3(3.4)$ & $1(5.9)$ & 0.516 & $<0.001$ \\
\hline Chronic kidney disease & $62(13.5)$ & $50(12.7)$ & $12(18.2)$ & 0.243 & $3(2.9)$ & $2(2.3)$ & $1(5.9)$ & 0.418 & 0.001 \\
\hline Cerebrovascular disease & $88(19.1)$ & $74(18.8)$ & $14(21.2)$ & 0.615 & $15(14.4)$ & $14(16.1)$ & $1(5.9)$ & 0.456 & 0.325 \\
\hline Current smoking & $110(23.9)$ & $97(24.6)$ & $13(19.7)$ & 0.438 & $43(41.3)$ & $40(46.0)$ & $3(17.6)$ & 0.034 & $<0.001$ \\
\hline $\mathrm{TSH}, \mathrm{mIU} / \mathrm{L}$ & $2.45 \pm 1.79$ & $1.91 \pm 0.90$ & $5.7 \pm 2.3$ & $<0.001$ & $2.61 \pm 1.52$ & $2.09 \pm 0.91$ & $5.32 \pm 1.09$ & $<0.001$ & 0.398 \\
\hline Free $\mathrm{T} 4, \mathrm{ng} / \mathrm{dL}$ & $1.15 \pm 0.22$ & $1.15 \pm 0.22$ & $1.15 \pm 0.23$ & 0.896 & $1.22 \pm 0.23$ & $1.24 \pm 0.22$ & $1.14 \pm 0.29$ & 0.132 & 0.005 \\
\hline Total T3, ng/dL & $87.7 \pm 42.4$ & $81.7 \pm 45.5$ & $97.8 \pm 38.6$ & 0.003 & $83.4 \pm 52.2$ & $78.8 \pm 55.8$ & $82.1 \pm 44.5$ & 0.792 & 0.449 \\
\hline Hemoglobin, g/dL & $13.0 \pm 2.0$ & $13.1 \pm 2.0$ & $12.6 \pm 2.2$ & 0.062 & $12.8 \pm 2.0$ & $12.9 \pm 1.9$ & $12.5 \pm 2.4$ & 0.457 & 0.491 \\
\hline Fasting glucose, $\mathrm{mg} / \mathrm{dL}$ & $65.7 \pm 66.8$ & $66.2 \pm 66.2$ & $62.9 \pm 70.9$ & 0.713 & $35.8 \pm 49.7$ & $35.9 \pm 50.0$ & $35.3 \pm 49.8$ & 0.962 & $<0.001$ \\
\hline $\mathrm{HbA1c}, \%$ & $5.0 \pm 2.7$ & $5.0 \pm 3.4$ & $5.5 \pm 3.2$ & 0.215 & $2.7 \pm 3.1$ & $2.7 \pm 3.1$ & $2.6 \pm 3.4$ & 0.909 & $<0.001$ \\
\hline Total cholesterol, mg/dL & $177.3 \pm 40.0$ & $177.2 \pm 39.2$ & $178.0 \pm 45.1$ & 0.880 & $179.5 \pm 45.5$ & $176.8 \pm 41.4$ & $192.8 \pm 62.5$ & 0.187 & 0.626 \\
\hline LDL-C, mg/dL & $68.3 \pm 52.4$ & $68.8 \pm 51.8$ & $65.1 \pm 56.0$ & 0.594 & $55.1 \pm 53.5$ & $52.9 \pm 51.7$ & $66.5 \pm 61.2$ & 0.338 & 0.021 \\
\hline HDL-C, mg/dL & $39.3 \pm 19.0$ & $38.8 \pm 9.2$ & $42.1 \pm 17.7$ & 0.194 & $34.5 \pm 23.3$ & $34.6 \pm 22.4$ & $33.6 \pm 8.1$ & 0.868 & 0.049 \\
\hline Triglyceride, mg/dL & $126.0 \pm 91.2$ & $124.5 \pm 88.8$ & $134.9 \pm 104.9$ & 0.390 & $94.3 \pm 80.0$ & $97.6 \pm 80.5$ & $77.4 \pm 77.4$ & 0.342 & 0.001 \\
\hline $\mathrm{CRP}, \mathrm{mg} / \mathrm{dL}$ & $1.20 \pm 2.69$ & $1.18 \pm 2.78$ & $1.31 \pm 2.11$ & 0.737 & $0.86 \pm 1.53$ & $0.88 \pm 1.55$ & $0.77 \pm 1.48$ & 0.808 & 0.259 \\
\hline Creatinine, $\mathrm{mg} / \mathrm{dL}$ & $1.42 \pm 1.31$ & $1.39 \pm 1.29$ & $1.62 \pm 1.39$ & 0.186 & $1.05 \pm 0.30$ & $1.04 \pm 0.27$ & $1.11 \pm 0.43$ & 0.366 & $<0.001$ \\
\hline $\begin{array}{l}\text { Values are expressed as m } \\
\text { IHD, ischemic heart disea } \\
\text { T4, thyroxine; T3, triiodot } \\
\text { CRP, C-reactive protein. }\end{array}$ & onine; $\mathrm{HbA}$ & eviation or num & tber $(\%)$ & w-densit & retain & HDI & igh-densi & 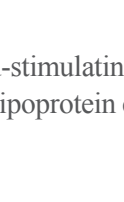 & olestero \\
\hline
\end{tabular}

Table 2. Long-Term Cardiovascular Outcomes According to Thyroid Function Status

\begin{tabular}{|c|c|c|c|c|c|c|}
\hline \multirow{2}{*}{ Outcome } & \multicolumn{3}{|c|}{ Patients with IHD } & \multicolumn{3}{|c|}{ Patients with VHD } \\
\hline & Euthyroid $(n=395)$ & $\mathrm{SCH}(n=66)$ & $P$ value & Euthyroid $(n=87)$ & $\mathrm{SCH}(n=17)$ & $P$ value \\
\hline All-cause mortality & $120(30.3)$ & $36(54.5)$ & $<0.001$ & $25(28.7)$ & $6(35.3)$ & 0.575 \\
\hline Cardiovascular mortality & $60(15.2)$ & $20(30.3)$ & 0.002 & $15(17.2)$ & $2(11.8)$ & 0.732 \\
\hline MACE & $84(21.2)$ & $18(27.2)$ & 0.260 & $23(26.4)$ & $2(11.8)$ & 0.351 \\
\hline Hospitalization for heart failure & $20(5.1)$ & $4(6.1)$ & 0.761 & $6(6.9)$ & $0(0.0)$ & 0.666 \\
\hline Fatal or nonfatal stroke & $46(11.6)$ & $11(16.7)$ & 0.104 & $12(13.8)$ & $1(5.9)$ & 0.457 \\
\hline Coronary revascularization & $27(6.8)$ & $5(7.6)$ & 0.792 & $1(1.1)$ & $0(0.0)$ & 1.000 \\
\hline
\end{tabular}

Values are expressed as number (\%). $P$ values were analyzed by the log-rank test.

IHD, ischemic heart disease; VHD, valvular heart disease; SCH, subclinical hypothyroidism; MACE, major adverse cardiovascular events.

CABG and HVS groups, underlying diseases and laboratory parameters also did not show statistically significant differences according to thyroid function status (i.e., between euthyroid and $\mathrm{SCH}$ patients). Serum TSH levels were significantly higher in 
SCH patients than in euthyroid patients, while fT4 levels were not significantly different according to thyroid function status in either the CABG or HVS group (Table 1).

\section{Long-term cardiovascular prognosis}

During a mean follow-up duration of $7.6 \pm 3.8$ years, in the CABG group, there were 156 (33.8\%) all-cause deaths, 80 (17.4\%) cardiovascular deaths, 112 (24.3\%) MACEs, 20 (4.3\%) hospitalizations for heart failure, 91 (19.7\%) cases of postoperative Afib, 32 (6.9\%) coronary revascularizations, 11 (2.4\%) MIs, and 57 (12.4\%) strokes (Table 2). During the same follow-up period, in the HVS group, there were 31 all-cause deaths $(29.8 \%$, $P=0.489$ vs. CABG group), 17 cardiovascular deaths (16.3\%, $P=0.886$ vs. CABG group), 25 MACEs $(24.0 \%, P=0.697$ vs. $\mathrm{CABG}$ group), six hospitalizations for heart failure $(5.8 \%, P=$ 0.809 vs. CABG group), 10 cases of postoperative Afib (9.6\%, $P=0.022$ vs. $\mathrm{CABG}$ group), one coronary revascularization ( $1.0 \%, P=0.007$ vs. CABG group), 0 MIs ( $0.0 \%, P=0.230$ vs. CABG group), and 13 strokes ( $12.5 \%, P=1.000$ vs. CABG group). Except for postoperative Afib and stroke, poor outcomes were more common in the CABG group than in the HVS group. When we compared those outcomes between $\mathrm{SCH}$ and euthyroid patients, the incidence of all-cause mortality $(55.4 \%$ vs. $30.3 \%, \log$-rank $P<0.001)$ and cardiovascular mortality (30.8\% vs. $15.2 \%$, log-rank $P<0.001)$ was higher in the $\mathrm{SCH}$ patients in the CABG group. The other outcomes, including MACE, hospitalization for heart failure, stroke, and coronary revascularization occurred more frequently in $\mathrm{SCH}$ patients than in euthyroid patients in the CABG group, but the differences were not significant (Table 2). Contrary to the outcomes of the CABG group, thyroid function status showed no significant relationships with the incidence of all-cause mortality, cardiovascular mortality, MACE, hospitalization for heart failure, stroke, or coronary revascularization in HVS patients (Table 2).

\section{Multivariate analysis of the long-term prognosis of SCH patients}

In the $\mathrm{CABG}$ group, $\mathrm{SCH}$ patients showed a significantly higher incidence of all-cause mortality than euthyroid patients (HR, $1.95 ; 95 \%$ CI, 1.29 to $2.93 ; P=0.002$ ) (Table 3, Fig. 1A). The incidence of cardiovascular mortality was also significantly higher in $\mathrm{SCH}$ patients than in euthyroid patients (HR, 2.30; $95 \%$ CI, 1.29 to $4.12 ; P=0.005$ ). (Table 3, Fig. 1B).

After adjustment for age and sex (model 1), the HR of allcause death after CABG was significantly higher in $\mathrm{SCH}$ patients than in euthyroid patients (HR, 2.09; $95 \%$ CI, 1.38 to 3.17 ;
Table 3. Multivariate Analysis of Hazard Ratios in Subclinical Hypothyroidism Patients Relative to Euthyroid Patients with Ischemic Heart Disease

\begin{tabular}{|c|c|c|c|}
\hline Variable & HR & $95 \% \mathrm{CI}$ & $P$ value \\
\hline \multicolumn{4}{|c|}{ All-cause mortality } \\
\hline Unadjusted & 1.95 & $1.29-2.93$ & 0.002 \\
\hline Model 1 & 2.09 & $1.38-3.17$ & $<0.001$ \\
\hline Model 2 & 1.93 & $1.27-2.93$ & 0.002 \\
\hline Model 3 & 1.70 & $1.10-2.63$ & 0.018 \\
\hline \multicolumn{4}{|c|}{ Cardiovascular mortality } \\
\hline Unadjusted & 2.30 & $1.29-4.12$ & 0.005 \\
\hline Model 1 & 2.43 & $1.35-4.37$ & 0.003 \\
\hline Model 2 & 2.25 & $1.24-4.07$ & 0.007 \\
\hline Model 3 & 1.95 & $1.05-3.61$ & 0.035 \\
\hline \multicolumn{4}{|l|}{ MACE } \\
\hline Unadjusted & 1.43 & $0.77-2.64$ & 0.258 \\
\hline Model 1 & 1.50 & $0.80-2.78$ & 0.203 \\
\hline Model 2 & 1.46 & $0.78-2.73$ & 0.235 \\
\hline Model 3 & 1.33 & $0.69-2.55$ & 0.391 \\
\hline \multicolumn{4}{|c|}{ Hospitalization for heart failure } \\
\hline Unadjusted & 1.54 & $0.52-4.55$ & 0.431 \\
\hline Model 1 & 1.66 & $0.56-4.92$ & 0.359 \\
\hline Model 2 & 1.59 & $0.53-4.79$ & 0.408 \\
\hline Model 3 & 1.18 & $0.34-4.13$ & 0.795 \\
\hline \multicolumn{4}{|c|}{ Fatal or nonfatal stroke } \\
\hline Unadjusted & 1.27 & $0.54-3.02$ & 0.586 \\
\hline Model 1 & 1.23 & $0.52-2.95$ & 0.639 \\
\hline Model 2 & 1.23 & $0.51-2.96$ & 0.653 \\
\hline Model 3 & 1.21 & $0.50-2.93$ & 0.674 \\
\hline \multicolumn{4}{|c|}{ Coronary revascularization } \\
\hline Unadjusted & 1.39 & $0.54-3.63$ & 0.497 \\
\hline Model 1 & 1.30 & $0.50-3.41$ & 0.595 \\
\hline Model 2 & 1.32 & $0.50-3.52$ & 0.577 \\
\hline Model 3 & 1.58 & $0.59-4.19$ & 0.362 \\
\hline
\end{tabular}

Multivariable Cox regression analysis was done. Model 1 adjusted for age and sex. Model 2 adjusted for the factors in model 1 and additionally adjusted for body mass index, smoking status, history of diabetes, and hypertension, dyslipidemia, and chronic kidney disease. Model 3 adjusted for the factors in model 2 and additionally adjusted for left ventricular dysfunction (ejection fraction $<40 \%$ ) and emergent surgery. $\mathrm{HR}$, hazard ratio; CI, confidence interval; MACE, major adverse cardiovascular events.

$P<0.001)$. We observed that the risk of all-cause death in model 2 (adjusting for BMI, smoking status, and the underlying diseases previously mentioned in addition to the variables in model 1) was significantly higher in $\mathrm{SCH}$ patients than in euthyroid pa- 


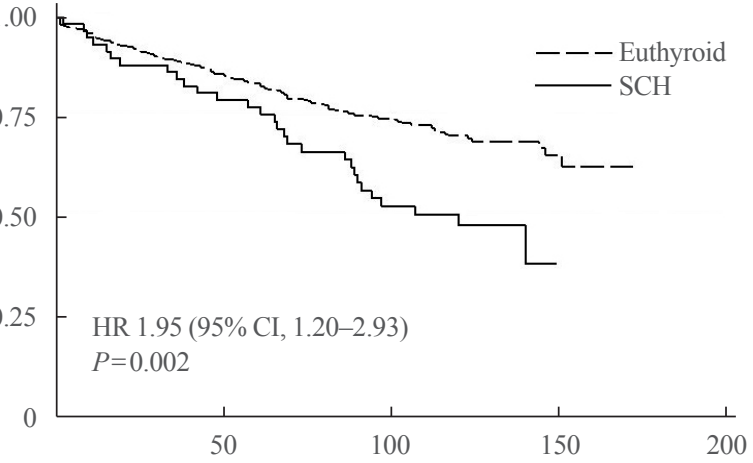

Number at risk

$$
\text { Follow-up duration (mo) }
$$

Euthyroid 382

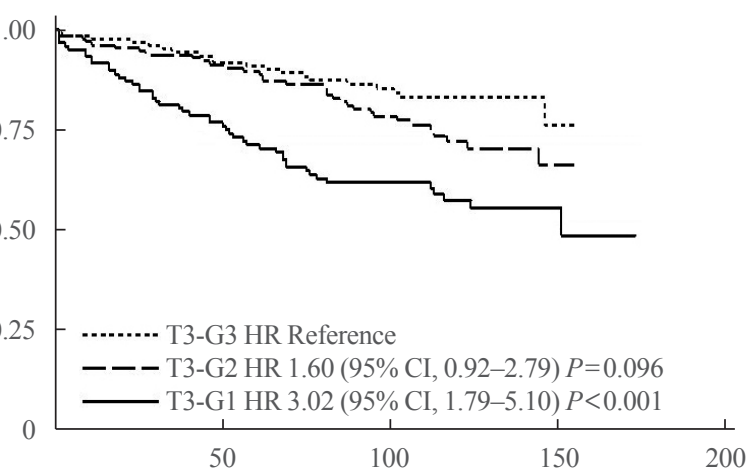

Number at risk

$$
\text { Follow-up duration (mo) }
$$

\section{0}

T3-G3 117

T3-G2 128

T3-G1 124

$\begin{array}{rrrr}82 & 55 & 8 & 0 \\ 109 & 78 & 7 & 0 \\ 82 & 55 & 8 & 0\end{array}$
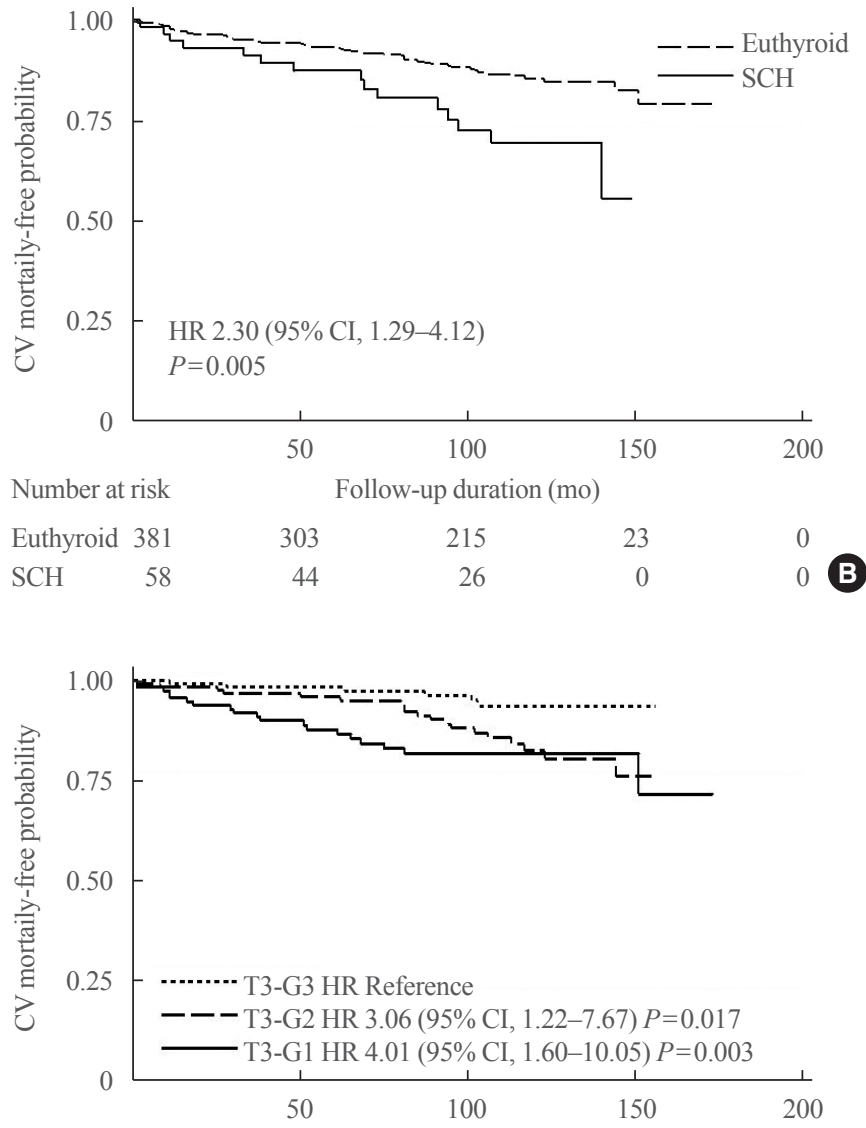

Number at risk

T3-G3 116

T3-G2 128

C $\quad \mathrm{T} 3-\mathrm{G} 1 \quad 124$
Follow-up duration (mo)

$\begin{array}{rrrr}82 & 55 & 8 & 0 \\ 109 & 78 & 7 & 0 \\ 104 & 75 & 7 & 0\end{array}$

0
0
0

Fig. 1. Kaplan-Meier curves of cardiovascular outcomes in patients with ischemic heart disease. (A) All-cause mortality in subclinical hypothyroidism ( $\mathrm{SCH}$ ) patients over euthyroid patients. (B) Cardiovascular (CV) mortality in SCH patients over euthyroid patients. (C) All-cause mortality according to the total triiodothyronine (T3) tertile in euthyroid patients. (D) Cardiovascular mortality according to the total T3 tertile in euthyroid patients. Cox regression analysis was performed. The hazard ratios (HRs) are for SCH patients with euthyroid patients as references. The HRs are for T3-G1 and T3-G2 patients with T3-G3 as reference. Total T3 tertiles: T3-G1, 0.3 to 83 ng/dL; T3-G2, 85 to 109 ng/dL; T3-G3, 110 to 163 ng/dL. CI, confidence interval.

tients (HR, $1.93 ; 95 \% \mathrm{CI}, 1.27$ to $2.93 ; P=0.002)$. After adjusting for the variables in model 3 (model 2 plus left ventricular dysfunction and emergent surgery), the risk of all-cause deaths after CABG also remained higher in $\mathrm{SCH}$ patients than in euthyroid patients (HR, $1.70 ; 95 \% \mathrm{CI}, 1.10$ to $2.63 ; P=0.018$ ). Interestingly, the HR of cardiovascular mortality for $\mathrm{SCH}$ patients relative to euthyroid patients who underwent $\mathrm{CABG}$ showed a similar tendency after adjustment for variables. In contrast, the HRs of MACE, hospitalization for heart failure, strokes, and coronary revascularization were not significantly different with and without adjustment for variables (Table 3).

In HVS patients, the HRs of all-cause mortality, cardiovascu- lar mortality, MACE and stroke in SCH patients relative to euthyroid patients did not significantly increase before and after adjustment for variables (Supplemental Table S1, Supplemental Fig. S1).

\section{Subgroup analysis of long-term cardiovascular prognosis in euthyroid subjects}

We conducted a subgroup analysis of euthyroid patients to elucidate the specific effects of thyroid function on postoperative outcomes after heart surgery. We divided euthyroid patients into three groups (G1, the lowest tertile; G2, the middle tertile; G3, the highest tertile) according to total T3, fT4, and TSH levels. 
Table 4. Long-Term Cardiovascular Outcomes According to Total T3 Tertiles in Euthyroid Patients

\begin{tabular}{|c|c|c|c|c|c|c|c|c|}
\hline \multirow[b]{2}{*}{ Outcome } & \multicolumn{4}{|c|}{ Patients with IHD $(n=395)$} & \multicolumn{4}{|c|}{ Patients with VHD ( $n=87)$} \\
\hline & $\begin{array}{c}\text { T3-G1 } \\
(n=125)\end{array}$ & $\begin{array}{c}\mathrm{T} 3-\mathrm{G} 2 \\
(n=130)\end{array}$ & $\begin{array}{c}\text { T3-G3 } \\
(n=126)\end{array}$ & $P$ value & $\begin{array}{l}\text { T3-G1 } \\
(n=27)\end{array}$ & $\begin{array}{l}\mathrm{T} 3-\mathrm{G} 2 \\
(n=27)\end{array}$ & $\begin{array}{l}\text { T3-G3 } \\
(n=27)\end{array}$ & $P$ value \\
\hline All-cause mortality & $59(45.7)$ & $32(25.4)$ & $23(18.1)$ & $<0.001$ & $6(22.2)$ & $7(29.6)$ & $3(25.9)$ & 0.729 \\
\hline Cardiovascular mortality & $29(22.5)$ & $19(15.1)$ & $8(6.3)$ & $<0.001$ & $5(18.5)$ & $4(14.8)$ & $4(14.8)$ & 0.998 \\
\hline MACE & $35(27.1)$ & $28(22.2)$ & $17(13.4)$ & 0.005 & $8(29.6)$ & $3(11.1)$ & $10(37.0)$ & 0.120 \\
\hline Hospitalization for heart failure & $11(8.5)$ & $6(4.8)$ & $3(2.4)$ & 0.083 & $3(11.1)$ & $1(3.7)$ & $2(7.4)$ & 0.791 \\
\hline Fatal or nonfatal stroke & $17(13.2)$ & $15(11.9)$ & $12(9.4)$ & 0.277 & $3(11.1)$ & $2(7.4)$ & $6(22.2)$ & 0.168 \\
\hline Coronary revascularization & $9(7.0)$ & $11(8.7)$ & $7(5.5)$ & 0.549 & $1(3.7)$ & $0(0.0)$ & $0(0.0)$ & 0.375 \\
\hline
\end{tabular}

Values are expressed as number (\%). $P$ values were analyzed by the log-rank test. Total T3 tertiles in patients with IHD: T3-G1, 0.3 to 83 ng/dL; T3-G2, 85 to $109 \mathrm{ng} / \mathrm{dL}$; T3-G3, 110 to $163 \mathrm{ng} / \mathrm{dL}$. Total T3 tertiles in patients with VHD: T3-G1, 0.3 to $80 \mathrm{ng} / \mathrm{dL}$; T3-G2, 83 to $118 \mathrm{ng} / \mathrm{dL}$; T3-G3, 119 to 167 $\mathrm{ng} / \mathrm{dL}$.

T3, triiodothyronine; IHD, ischemic heart disease; VHD, valvular heart disease; MACE, major adverse cardiovascular events.

In CABG patients, the incidence of all-cause mortality, cardiovascular mortality, and MACE significantly increased from the highest total T3 tertile (T3-G3) to the lowest total T3 tertile (T3-G1) (log-rank test $P<0.001, P<0.001$, and $P=0.005$, respectively) (Table 4, Fig. 1C, D). However, the incidence of hospitalization for heart failure, stroke and coronary revascularization did not change as the total T3 level decreased (Table 4). On the contrary, there was no correlation between total T3 levels and the incidence of long-term cardiovascular outcomes in euthyroid patients after HVS (Table 4, Supplemental Fig. S2).

We conducted multivariate analysis according to the total T3 tertile in euthyroid patients with ischemic heart disease. Interestingly, the HR of all-cause mortality in the T3-G1 tertile patients was significantly higher, by over 3 -fold, than that in the T3-G3 tertile group before adjustment $(P<0.001)$. After adjustment in model 1 and model 2, the HRs of the T3-G1 tertile were 2.84 (95\% CI, 1.68 to $4.80 ; P<0.001)$ and $1.98(95 \%$ CI, 1.13 to 3.46 ; $P=0.017)$, respectively. However, statistical significance disappeared in model 3 (Table 5). More notably, cardiovascular mortality in the T3-G1 tertile group was more than 4-fold higher than in the T3-G3 tertile patients after CABG, with an HR of 4.01 (95\% CI, 1.60 to $10.05 ; P=0.003$ ). Furthermore, the T3-G2 tertile patients showed a higher cardiovascular mortality rate, by over 3-fold, than the T3-G3 tertile patients (HR, 3.06; 95\% CI, 1.22 to $7.67 ; P=0.017$ ). Moreover, the risk of cardiovascular mortality in the T3-G1 and T3-G2 tertile patients remained significantly higher (at least 2-fold) than in the T3-G3 tertile after adjustment in model 1 and 2. However, statistical significance disappeared in model 3 (Table 5). The risk of hospitalization for heart failure in the T3-G1 tertile was more than five times higher than that in the T3-T3 tertile, although the HR was not significant after adjustment. In contrast, the incidence of MACE, stroke, and coronary revascularization did not increase with decreasing total $\mathrm{T} 3$ levels (Table 5).

However, the long-term outcomes were not significantly affected by the fT4 tertile or TSH tertile in the CABG group or the HVS group (Supplemental Table S2, Supplemental Figs. S3, S4).

\section{DISCUSSION}

In this study, during the mean follow-up period of 7.6 years, preoperative $\mathrm{SCH}$ patients showed higher all-cause mortality and cardiovascular mortality after CABG than their euthyroid counterparts. The HR for all-cause mortality was 1.70 after adjustment for covariates. Cardiovascular mortality was also significantly higher, by 1.94 -fold, in patients with preoperative SCH after CABG. Additionally, we found that patients with lower total T3 levels — even with euthyroid status — showed significantly higher risks for all-cause and cardiovascular death after CABG. Notably, all these associations were only observed in patients with ischemic heart disease who had high cardiovascular risk, but not in patients with valvular heart disease. These results suggest that preoperative $\mathrm{SCH}$ and low total $\mathrm{T} 3$ in euthyroid patients are important factors associated with long-term outcomes after CABG.

Some results have provided support for the possibility that $\mathrm{SCH}$ is a risk factor for CHD. Rodondi et al. [12] reported that $\mathrm{SCH}$ was associated with an increased risk of CHD in their meta-analysis. They also showed a significant positive correlation between TSH elevation and increased mortality from CHD in 
Table 5. Multivariate Analysis of Hazard Ratios in Euthyroid Patients with Ischemic Heart Disease According to Total T3 Tertiles

\begin{tabular}{|c|c|c|c|c|c|c|c|c|}
\hline \multirow{2}{*}{ Variable } & \multicolumn{2}{|c|}{ Univariate } & \multicolumn{2}{|c|}{ Model 1} & \multicolumn{2}{|c|}{ Model 2} & \multicolumn{2}{|l|}{ Model 3} \\
\hline & $\mathrm{HR}(95 \% \mathrm{CI})$ & $P$ value & $\mathrm{HR}(95 \% \mathrm{CI})$ & $P$ value & $\mathrm{HR}(95 \% \mathrm{CI})$ & $P$ value & $\mathrm{HR}(95 \% \mathrm{CI})$ & $P$ value \\
\hline \multicolumn{9}{|c|}{ All-cause mortality } \\
\hline T3-G1 & $3.02(1.79-5.10)$ & $<0.001$ & $2.84(1.68-4.80)$ & $<0.001$ & $1.98(1.13-3.46)$ & 0.017 & $1.67(0.94-2.97)$ & 0.079 \\
\hline T3-G2 & $1.60(0.92-2.79)$ & 0.096 & $1.61(0.92-2.82)$ & 0.097 & $1.17(0.65-2.10)$ & 0.592 & $1.03(0.57-1.89)$ & 0.912 \\
\hline T3-G3 & Reference & & Reference & & Reference & & Reference & \\
\hline \multicolumn{9}{|c|}{ Cardiovascular mortality } \\
\hline T3-G1 & $4.01(1.60-10.05)$ & 0.003 & $3.85(1.53-9.68)$ & 0.004 & $3.32(1.26-8.79)$ & 0.016 & $2.47(0.92-6.65)$ & 0.073 \\
\hline T3-G2 & $3.06(1.22-7.67)$ & 0.017 & $3.04(1.21-7.68)$ & 0.019 & $2.68(1.03-7.02)$ & 0.044 & $2.05(0.77-5.48)$ & 0.151 \\
\hline T3-G3 & Reference & & Reference & & Reference & & Reference & \\
\hline
\end{tabular}

MACE

\begin{tabular}{ccccccccc} 
T3-G1 & $1.10(0.62-1.95)$ & 0.745 & $1.12(0.63-2.02)$ & 0.695 & $1.15(0.62-2.11)$ & 0.664 & $1.20(0.65-2.23)$ & 0.562 \\
T3-G2 & $0.73(0.39-1.36)$ & 0.325 & $0.72(0.39-1.35)$ & 0.306 & $0.67(0.36-1.27)$ & 0.224 & $0.71(0.37-1.35)$ & 0.300 \\
T3-G3 & Reference & \multicolumn{3}{c}{ Reference } & Reference & Reference
\end{tabular}

Hospitalization for heart failure

$\begin{array}{lcccccccc}\text { T3-G1 } & 5.09(1.14-22.80) & 0.033 & 4.04(0.34-9.35) & 0.069 & 4.05(0.82-20.08) & 0.087 & 3.80(0.74-19.44) & 0.109 \\ \text { T3-G2 } & 1.91(0.37-9.86) & 0.439 & 1.79(0.90-18.15) & 0.489 & 1.78(0.32-9.96) & 0.511 & 1.89(0.33-10.74) & 0.472 \\ \text { T3-G3 } & \text { Reference } & \text { Reference } & & \text { Reference } & \text { Reference }\end{array}$

Fatal or nonfatal stroke

$\begin{array}{lccccccccc}\text { T3-G1 } & 1.40(0.59-3.30) & 0.441 & 1.44(0.61-3.41) & 0.403 & 1.34(0.53-3.44) & 0.536 & 1.17(0.45-3.07) & 0.748 \\ \text { T3-G2 } & 1.24(0.54-2.82) & 0.612 & 1.21(0.53-2.76) & 0.653 & 1.25(0.53-2.96) & 0.608 & 0.96(0.39-2.37) & 0.937 \\ \text { T3-G3 } & \text { Reference } & & \text { Reference } & & \text { Reference } & \text { Reference } \\ \text { Coronary revascularization } & & & & & & \\ \text { T3-G1 } & 1.67(0.66-4.25) & 0.279 & 1.59(0.62-4.10) & 0.337 & 1.21(0.44-3.28) & 0.711 & 1.57(0.56-4.45) & 0.393 \\ \text { T3-G2 } & 1.01(0.37-2.78) & 0.987 & 1.00(0.36-2.75) & 0.992 & 0.82(0.29-2.33) & 0.709 & 1.00(0.34-2.99) & 0.993 \\ \text { T3-G3 } & \text { Reference } & & \text { Reference } & & \text { Reference } & \text { Reference }\end{array}$

Multivariable Cox regression analysis was done. The hazard ratios are for T3-G1 and T3-G2 participants with T3-G3 as reference. Model 1 adjusted for age and sex. Model 2 adjusted for the factors in model 1 and additionally adjusted for body mass index, smoking status, history of diabetes and hypertension, dyslipidemia, and chronic kidney disease. Model 3 adjusted for the factors in model 2 and additionally adjusted for left ventricular dysfunction (ejection fraction $<40 \%$ ) and emergent surgery. Total T3 tertiles: T3-G1, 0.3 to $83 \mathrm{ng} / \mathrm{dL}$; T3-G2, 85 to $109 \mathrm{ng} / \mathrm{dL}$; T3-G3, 110 to $163 \mathrm{ng} / \mathrm{dL}$.

T3, triiodothyronine; HR, hazard ratio; CI, confidence interval; MACE, major adverse cardiovascular events.

another meta-analysis of 55,287 adults, particularly in those with a TSH concentration of $10 \mathrm{mIU} / \mathrm{L}$ or greater [12]. Similarly, elevated serum TSH levels significantly increased the risk of CHD by approximately $20 \%$ per one standard deviation in men in a Korean cohort followed for 10 years [24].

We previously showed that $\mathrm{SCH}$ was associated with postoperative Afib within 3 months after CABG [16], and that postoperative Afib in $\mathrm{SCH}$ patients after $\mathrm{CABG}$ was related to an increased risk of poor postoperative outcomes, particularly coronary revascularization [17]. Although the mechanism is unclear, TSH itself has been suggested to stimulate the proliferation of vascular smooth muscle cells, endothelial cell damage, and in- flammation, leading to atherosclerosis [25-27]. Patients with SCH also showed increased levels of inflammatory cytokines, lymphocytes, macrophages, and nitrotyrosine, reflecting endothelial dysfunction, but decreased collagen content in vascular plaques [27]. In this regard, a previous study suggested that patients with $\mathrm{SCH}$ are susceptible to atherogenesis, which may lead to restenosis.

Importantly, we found that $\mathrm{CABG}$ patients with $\mathrm{SCH}$ were at an increased risk of all-cause death and cardiovascular death during the follow-up period. An association between mortality and $\mathrm{SCH}$ in $\mathrm{CABG}$ patients was not observed in our prior studies $[16,17]$. This discrepancy might reflect the increased number 
of subjects and the data retrieval method. We were able to analyze about twice as many subjects by including new patients, although it resulted in a relatively short follow-up duration of $7.5 \pm 3.9$ years, compared to the follow-up period of $8.2 \pm 4.1$ years in our prior study. Moreover, in this study, we collected patients' mortality records of the National Statistical Office in South Korea in addition to the medical records of SNUBH. Thus, the mortality rate and the cause of death might have been more accurate than in previous studies, and we were able to demonstrate that $\mathrm{SCH}$ increased the risk of mortality in $\mathrm{CABG}$ patients. Another important finding was that the increased risk was only observed in patients with a higher risk of CHD. In HVS patients, the mortality rates were similar regardless of TSH levels, providing further supporting that the risk associated with higher TSH levels was not related to cardiac surgery, but to conditions associated with high cardiovascular risk. Regarding the associations of thyroid dysfunction in patients undergoing HVS, Martinez-Comendador et al. [18] reported that SCH was associated with an increased risk of postoperative Afib in patients undergoing aortic valve replacement, but there were limited data on the long-term outcomes of HVS patients according to thyroid function. We did not find any significant effects of $\mathrm{SCH}$ on postoperative outcomes in the HVS group, although the number of subjects might not have been sufficient to fully elucidate the impact of $\mathrm{SCH}$ on the long-term prognosis in patients with HVS.

In the subgroup analysis of euthyroid subjects, there were no significant differences in long-term outcomes after CABG and HVS according to the fT4 tertile, whereas the lowest T3 tertile showed an increased risk of all-cause mortality and cardiovascular mortality, by up to 3- or 4- fold compared with the highest T3 tertile after adjustment in model 1 and 2. Daswani et al. [28] showed that the occurrence of CHD was associated with lower serum free T3 (fT3) levels in the absence of primary thyroid diseases and acute coronary syndrome. In another Korean cohort study including euthyroid subjects $(n=212,456)$, fT4 and fT3 levels in euthyroid patients were inversely associated with the risks of all-cause and cancer mortality [29]. Although in this study, fT3 levels were not measured and fT4 levels did not show this tendency, the presence of preoperative lower total T3 levels, as well as $\mathrm{SCH}$, in CABG patients may be suggested as a poor prognostic factor affecting long-term cardiovascular outcomes. Furthermore, it is unclear whether the effects of SCH on longterm outcomes would be reduced by $\mathrm{T} 4$ replacement or whether T3 supplementation is needed instead of T4. More randomized controlled trials are needed to investigate this question conclu- sively.

This study has several strengths. The mortality and cause of death data were very accurate, because we collected them by double-checking the data of the National Statistical Office in South Korea and medical records of SNUBH. In addition, to the best of our knowledge, this study is the first to evaluate longterm cardiovascular outcomes according to $\mathrm{SCH}$ in patients undergoing HVS.

There are also potential limitations of this study. First, serial follow-up of postoperative thyroid function was not performed; therefore, we could not distinguish nonthyroidal illness before surgery from permanent thyroid dysfunction. Second, medical treatments affecting thyroid function such as amiodarone and iodine contrast might have been confounding factors in our study. Lastly, the number of patients with HVS might not have been sufficient to confirm the effect of thyroid function on postoperative outcomes in this group. Further studies are warranted to confirm the effects of thyroid function after other types of cardiac surgery, including HVS.

In conclusion, $\mathrm{SCH}$ and low total $\mathrm{T} 3$ levels in euthyroid patients were associated with an increased risk of long-term mortality after $\mathrm{CABG}$ in patients with ischemic heart disease, in contrast to HVS patients, who did not show such a tendency. Preoperative evaluations of thyroid function may be a meaningful marker for long-term cardiovascular outcomes in CABG patients.

\section{CONFLICTS OF INTEREST}

No potential conflict of interest relevant to this article was reported.

\section{ACKNOWLEDGMENTS}

The study was supported by grants from the Korea Health Industry Development Institute (HI17C1099) and Seoul National University Bundang Hospital research fund (No. 02-2012-058).

\section{AUTHOR CONTRIBUTIONS}

Conception or design: H.K., Y.J.P., C.L. Acquisition, analysis, or interpretation of data: H.K., S.H.K., J.H.M., S.Y.K., K.H.P., J.S.K., J.H.C., Y.J.P., C.L. Drafting the work or revising: H.K., Y.J.P., C.L. Final approval of the manuscript: H.K., Y.J.P., C.L. 


\section{ORCID}

Hana Kim https://orcid.org/0000-0002-9221-2988

Young Joo Park https://orcid.org/0000-0002-3671-6364

Cheong Lim https://orcid.org/0000-0003-0913-7014

\section{REFERENCES}

1. Kim YA, Park YJ. Prevalence and risk factors of subclinical thyroid disease. Endocrinol Metab (Seoul) 2014;29:20-9.

2. Park YJ, Lee YJ, Choi SI, Chun EJ, Jang HC, Chang HJ. Impact of subclinical hypothyroidism on the coronary artery disease in apparently healthy subjects. Eur J Endocrinol 2011;165:115-21.

3. Ceresini G, Ceda GP, Lauretani F, Maggio M, Usberti E, Marina M, et al. Thyroid status and 6-year mortality in elderly people living in a mildly iodine-deficient area: the aging in the Chianti Area Study. J Am Geriatr Soc 2013;61:86874.

4. Gussekloo J, van Exel E, de Craen AJ, Meinders AE, Frolich M, Westendorp RG. Thyroid status, disability and cognitive function, and survival in old age. JAMA 2004;292: 2591-9.

5. Pereg D, Tirosh A, Elis A, Neuman Y, Mosseri M, Segev D, et al. Mortality and coronary heart disease in euthyroid patients. Am J Med 2012;125:826.

6. Tseng FY, Lin WY, Lin CC, Lee LT, Li TC, Sung PK, et al. Subclinical hypothyroidism is associated with increased risk for all-cause and cardiovascular mortality in adults. J Am Coll Cardiol 2012;60:730-7.

7. Ittermann T, Haring R, Sauer S, Wallaschofski H, Dorr M, Nauck M, et al. Decreased serum TSH levels are not associated with mortality in the adult northeast German population. Eur J Endocrinol 2010;162:579-85.

8. Parle JV, Maisonneuve P, Sheppard MC, Boyle P, Franklyn JA. Prediction of all-cause and cardiovascular mortality in elderly people from one low serum thyrotropin result: a 10year cohort study. Lancet 2001;358:861-5.

9. Westerink J, van der Graaf Y, Faber DR, Spiering W, Visseren FL; SMART study group. Relation between thyroid-stimulating hormone and the occurrence of cardiovascular events and mortality in patients with manifest vascular diseases. Eur J Prev Cardiol 2012;19:864-73.

10. Hyland KA, Arnold AM, Lee JS, Cappola AR. Persistent subclinical hypothyroidism and cardiovascular risk in the elderly: the cardiovascular health study. J Clin Endocrinol
Metab 2013;98:533-40.

11. Selmer C, Olesen JB, Hansen ML, von Kappelgaard LM, Madsen JC, Hansen PR, et al. Subclinical and overt thyroid dysfunction and risk of all-cause mortality and cardiovascular events: a large population study. J Clin Endocrinol Metab 2014;99:2372-82.

12. Rodondi N, den Elzen WP, Bauer DC, Cappola AR, Razvi S, Walsh JP, et al. Subclinical hypothyroidism and the risk of coronary heart disease and mortality. JAMA 2010;304: 1365-74.

13. Ochs N, Auer R, Bauer DC, Nanchen D, Gussekloo J, Cornuz J, et al. Meta-analysis: subclinical thyroid dysfunction and the risk for coronary heart disease and mortality. Ann Intern Med 2008;148:832-45.

14. Moon S, Kong SH, Choi HS, Hwangbo Y, Lee MK, Moon $\mathrm{JH}$, et al. Relation of subclinical hypothyroidism is associated with cardiovascular events and all-cause mortality in adults with high cardiovascular risk. Am J Cardiol 2018; 122:571-7.

15. Moon S, Kim MJ, Yu JM, Yoo HJ, Park YJ. Subclinical hypothyroidism and the risk of cardiovascular disease and allcause mortality: a meta-analysis of prospective cohort studies. Thyroid 2018;28:1101-10.

16. Park YJ, Yoon JW, Kim KI, Lee YJ, Kim KW, Choi SH, et al. Subclinical hypothyroidism might increase the risk of transient atrial fibrillation after coronary artery bypass grafting. Ann Thorac Surg 2009;87:1846-52.

17. Kong SH, Yoon JW, Kim SY, Oh TJ, Park KH, Choh JH, et al. Subclinical hypothyroidism and coronary revascularization after coronary artery bypass grafting. Am J Cardiol 2018; 122:1862-70.

18. Martinez-Comendador J, Marcos-Vidal JM, Gualis J, Martin CE, Martin E, Otero J, et al. Subclinical hypothyroidism might increase the risk of postoperative atrial fibrillation after aortic valve replacement. Thorac Cardiovasc Surg 2016;64: 427-33.

19. O'Connor GT, Plume SK, Olmstead EM, Coffin LH, Morton JR, Maloney CT, et al. A regional prospective study of in-hospital mortality associated with coronary artery bypass grafting. The Northern New England Cardiovascular Disease Study Group. JAMA 1991;266:803-9.

20. Friedewald WT, Levy RI, Fredrickson DS. Estimation of the concentration of low-density lipoprotein cholesterol in plasma, without use of the preparative ultracentrifuge. Clin Chem 1972;18:499-502.

21. Ommen SR, Odell JA, Stanton MS. Atrial arrhythmias after 
cardiothoracic surgery. N Engl J Med 1997;336:1429-34.

22. Biondi B. Mechanisms in endocrinology: heart failure and thyroid dysfunction. Eur J Endocrinol 2012;167:609-18.

23. Galli E, Pingitore A, Iervasi G. The role of thyroid hormone in the pathophysiology of heart failure: clinical evidence. Heart Fail Rev 2010;15:155-69.

24. Kim TH, Choi HS, Bae JC, Moon JH, Kim HK, Choi SH, et al. Subclinical hypothyroidism in addition to common risk scores for prediction of cardiovascular disease: a 10-year community-based cohort study. Eur J Endocrinol 2014;171: 649-57.

25. Tian L, Ni J, Guo T, Liu J, Dang Y, Guo Q, et al. TSH stimulates the proliferation of vascular smooth muscle cells. Endocrine 2014;46:651-8.

26. Marfella R, Ferraraccio F, Rizzo MR, Portoghese M, Barb- ieri $\mathrm{M}$, Basilio $\mathrm{C}$, et al. Innate immune activity in plaque of patients with untreated and L-thyroxine-treated subclinical hypothyroidism. J Clin Endocrinol Metab 2011;96:1015-20.

27. Hassan A, Altamirano-Ufion A, Zulfiqar B, Boddu P. Subclinical hypothyroidism and its association with increased cardiovascular mortality: call for action. Cardiol Res 2017;8: 31-5.

28. Daswani R, Jayaprakash B, Shetty R, Rau NR. Association of thyroid function with severity of coronary artery disease in euthyroid patients. J Clin Diagn Res 2015;9:OC10-3.

29. Zhang Y, Chang Y, Ryu S, Cho J, Lee WY, Rhee EJ, et al. Thyroid hormones and mortality risk in euthyroid individuals: the Kangbuk Samsung health study. J Clin Endocrinol Metab 2014;99:2467-76. 